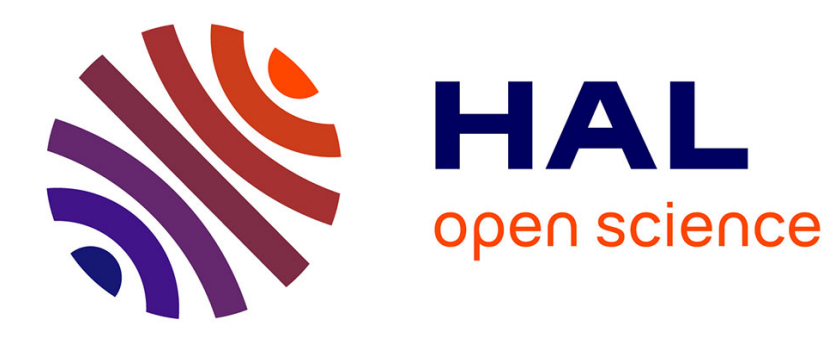

\title{
Overdetermined elliptic problems in physics
}

\author{
Boyan Sirakov
}

\section{To cite this version:}

Boyan Sirakov. Overdetermined elliptic problems in physics. 2002, pp.573. hal-00004765

\section{HAL Id: hal-00004765 \\ https://hal.science/hal-00004765}

Submitted on 20 Apr 2005

HAL is a multi-disciplinary open access archive for the deposit and dissemination of scientific research documents, whether they are published or not. The documents may come from teaching and research institutions in France or abroad, or from public or private research centers.
L'archive ouverte pluridisciplinaire HAL, est destinée au dépôt et à la diffusion de documents scientifiques de niveau recherche, publiés ou non, émanant des établissements d'enseignement et de recherche français ou étrangers, des laboratoires publics ou privés. 


\title{
Overdetermined elliptic problems in physics
}

\author{
Boyan SIRAKOV \\ Laboratoire MODALX, UFR SEGMI \\ Université Paris 10, Bâtiment G \\ 92001 Nanterre Cedex, FRANCE
}

\section{The physical problems}

In this survey we describe several physically motivated problems in which the corresponding mathematical models lead to overdetermined elliptic boundary-value problems. Such situations appear for instance in fluid mechanics and capillarity theory, theory of elasticity, electrostatics. Our goal here is to give an idea of how they have been treated mathematically.

\subsection{Interior overdetermined problems. Examples}

In this section we describe three physical problems which were first given mathematical consideration and solved by Serrin in his classical paper [21]. They lead to overdetermined elliptic boundary-value problems in bounded domains.

Fluid moving in a straight pipe. We begin with a simple example which will help us to introduce the kind of problems we consider. Suppose we have a viscous incompressible fluid moving in a straight pipe with a given cross section. Fix rectangular coordinates $(x, y, z)$ in space with the $z$-axis directed along the pipe. Then the cross section of the pipe containing the origin is a domain in the $(x, y)$-plane, which we denote by $\Omega$. It is a standard result from fluid mechanics that the flow velocity does not depend on $z$ and therefore can be regarded as a function of $x$ and $y$, defined in $\Omega$. Furthermore, it is known (see for example [12]) that $u$ satisfies the Poisson equation

$$
\Delta u=-\frac{\delta}{\eta l} \quad \text { in } \Omega,
$$

where $\eta$ denotes the dynamic viscosity, $l$ is the length of the pipe and $\delta$ is the change of pressure between the two ends of the pipe. 
The adherence condition on the wall of the pipe is expressed by the Dirichlet boundary condition

$$
u=0 \quad \text { on } \partial \Omega \text {. }
$$

Finally, the tangential stress on the wall is $\eta \frac{\partial u}{\partial n}$, where $n$ denotes the interior normal to $\partial \Omega$. The precise determination of the point of maximal tangential stress is an important but mathematically very difficult problem. Here we are interested in the following question : when is the tangential stress the same at each point of a cross section of the wall? In other words, can we have a solution of (1)-(2) which satisfies the Neumann type boundary condition

$$
\frac{\partial u}{\partial n}=\text { const } \quad \text { on } \partial \Omega ?
$$

It is very standard and classical to consider the Poisson equation (1) with either of the boundary conditions (2) or (3) and there is a huge literature on both problems (1)-(2) and (1)-(3). However, the question we asked above requires that both of these conditions be satisfied by the solution of (1) this is what we call overdetermined. Problem (1)-(2)-(3) can be viewed as a free boundary problem, in the sense that the domain is part of the problem.

It is intuitively clear that (2) and (3) together is too much to ask, and in most cases the answer to our question will be negative, that is, problem (1)-(2)-(3) will not be solvable. On the other hand, if $\Omega$ is a ball with radius $R$ then the unique solution of (1)-(2) is

$$
u(x)=\frac{\eta l}{4 \delta}\left(R^{2}-x^{2}-y^{2}\right),
$$

and hence satisfies (3). Thus a natural question to ask is whether there exists a non-circular pipe such that a fluid moving inside it has the same tangential stress on all points of its wall. Theorem 1 in Section 2 provides a negative answer to this question.

The torsion problem. An equation of type (1), together wtih the boundary condtions (2) and (3), arises when we model the torsion of a solid cylindrical bar. We follow the presentation in [23]. Suppose we have a cylindrical body of arbitrary (simply connected) cross section, one end of which is fixed, while the other is twisted by a couple of given magnitude. We fix the coordinate system as in the previous example, with the $z$-axis along the axis of the cylinder. It is known that, in general, after the bar is twisted its cross sections do not remain plane but are warped. Actually, any point $P(x, y, z)$ of the body occupies a new position $P^{\prime}(x+r, y+s, z+t)$ after the twisting, where

$$
r=-\alpha z y, \quad s=\alpha z x, \quad t=\alpha \varphi(x, y),
$$




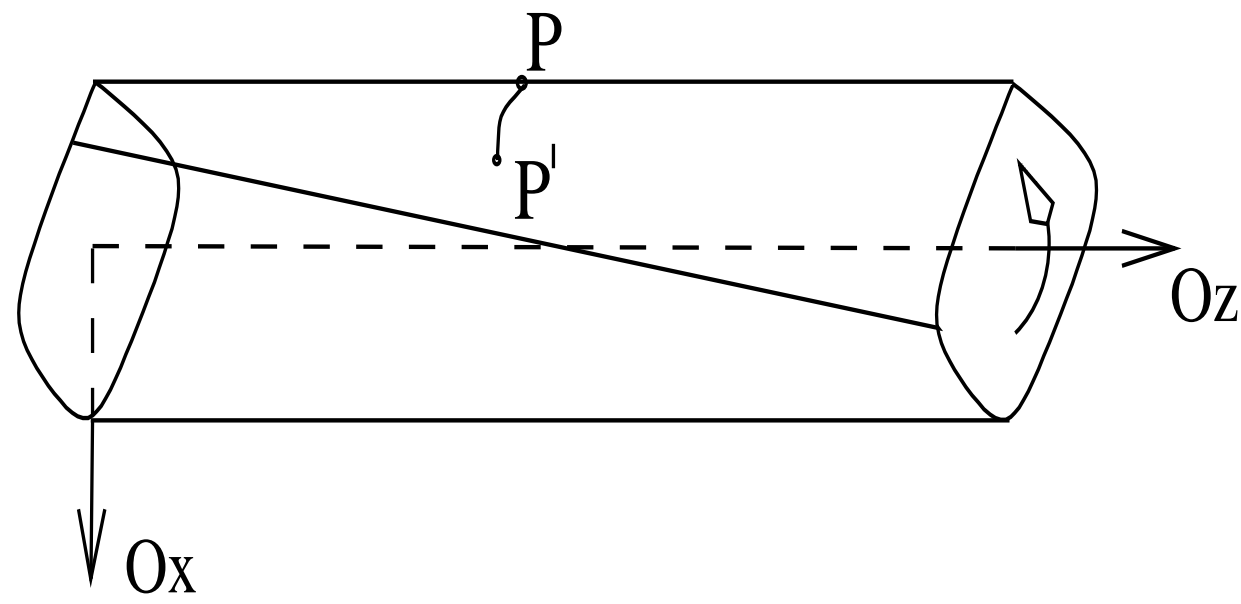

where $\alpha$ is the twist per unit length of the bar and $\varphi$ denotes the torsion function. Let us note that the torsion is zero (that is cross sections of the bar do remain plane) if and only if the cylinder is circular. This result turns out to be a very particular case of the symmetry theorems we present in Section 2.

We shall consider the torsion in terms of L. Prandtl's "stress function"

$$
\Psi(x, y)=\psi(x, y)-\frac{1}{2}\left(x^{2}+y^{2}\right)
$$

where $\psi(x, y)$ stands for the complex conjugate of $\varphi$. It can be checked ([23]) that $\Psi$ satisfies the equation

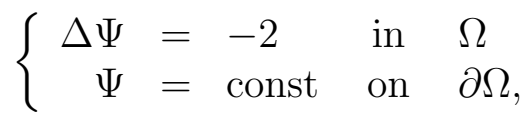

where $\Omega$ is an arbitrary cross section of the bar.

The function $\Psi$ has the following important property : at each point of a level curve of $\Psi$ (these curves, defined by $\Psi=$ const, are called lines of shearing stress) the stress vector is directed along the tangent to the curve. Furthermore, since all tangential derivatives of $\Psi$ are zero on $\partial \Omega$,

$$
\frac{\partial \Psi}{\partial n}=|\nabla \Psi|=\frac{1}{\mu \alpha} \cdot \tau \quad \text { on } \partial \Omega
$$

(see [23]) ; here $\mu$ denotes the modulus of rigidity of the bar and $\tau$ is the magnitude of tangential stress ( $\tau$ is called shearing stress).

Like in the previous example, the shearing stress is maximal on the lateral boundary, so elastic failure of the material is to be expected on that boundary. Here we address the following two questions : 
- Can the shearing stress be constant on the lateral surface?

- If the bar is invariant under some group action, in particular, if the bar is symmetric with respect to a hyperplane, then do lines of shearing stress have the same property?

The interior capillarity problem. A more complicated example is provided by the equation of equilibrium shapes of the surface of a homogeneous and incompressible liquid contained in a straight tube, subject to a gravitational field. Our presentation follows [15], Chapter 2.

We again consider a rectangular coordinate system with the $z$-axis directed along the axis of the vessel and denote with $\Omega$ the cross section of the tube containing the origin. For each $(x, y) \in \Omega$ we define $u(x, y)$ to be the height, with respect to the level of $\Omega$, to which the liquid rises above or below the point $(x, y)$.

In this situation, the first two conditions for hydrostatic equlibrium (Euler's condition and Laplace's condition) reduce to the following equation

$$
\operatorname{div} \frac{\nabla u}{\sqrt{1+|\nabla u|^{2}}}-b u=q \quad \text { in } \Omega,
$$

where $b=\frac{\rho g}{\sigma}$ and $q$ is some constant depending only on the height at which we fix the origin. As usual, $\rho$ denotes the density of the fluid, $\sigma$ is the surface tension, and $g$ is the intensity of the gravitational field. All these quantities are constants in this model.

In this setting the Dupré-Young condition for hydrostatic equilibrium becomes

$$
\frac{\partial u}{\partial n}=-\cos \alpha \sqrt{1+|\nabla u|^{2}} \quad \text { on } \partial \Omega,
$$

where $n$ is the interior normal to the boundary of $\partial \Omega$ and $\alpha$ is the contact (or wetting) angle between the liquid surface and the wall of the vessel.

The question we are interested in is : when does the liquid rise to the same height at each point of the wall ?

If $u=$ const on $\partial \Omega$ then the normal derivative of $u$ is equal to the length of the gradient of $u$ on $\partial \Omega$, so (7) transforms into

$$
\frac{\partial u}{\partial n}=-\operatorname{cotg} \alpha \quad \text { on } \partial \Omega .
$$

We shall exclude the two limiting cases $\alpha=0$ and $\alpha=\frac{\pi}{2}(\alpha=0$ is clearly irrealistic, while for $\alpha=\frac{\pi}{2}$ the only solution of (6)-(7) is $u \equiv \frac{-q}{b}$, independently of the shape of the vessel). 


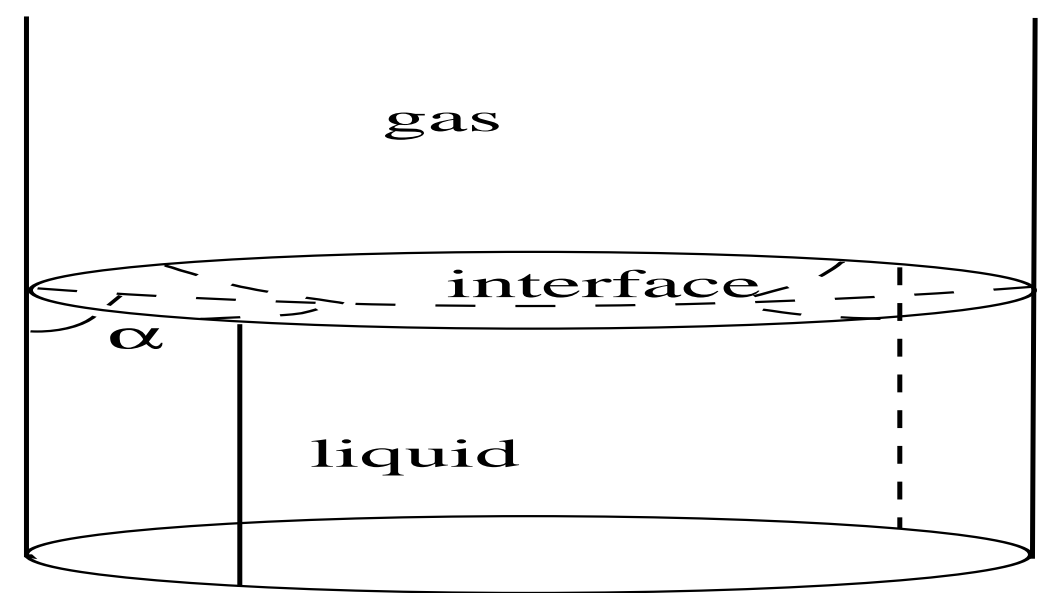

Note that the maximum principle (see Section 3), applied to (6), says $u$ attains its maximum in $\bar{\Omega}$ on the boundary $\partial \Omega$. By the strong maximum principle, if $u=$ const on $\partial \Omega$ then $u$ attains its maximum only on $\partial \Omega$, except if it is constant in $\Omega$. The latter is excluded by $\alpha \neq \frac{\pi}{2}$.

Finally, to answer the question we asked above, we have to study the solvability of the following problem

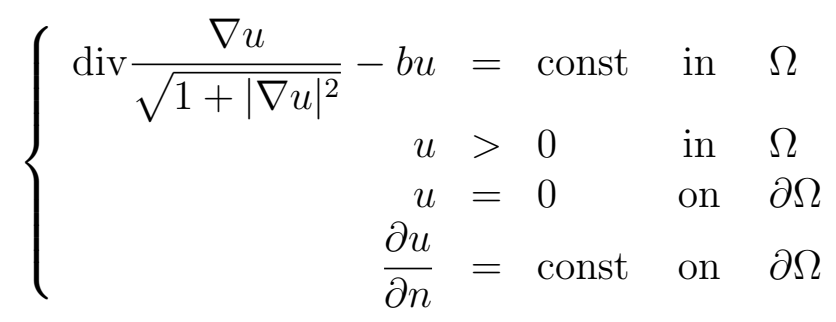

(we have fixed the reference level to be the surface level on the vessel wall, and have replaced $u$ by $-u$ ).

\subsection{Exterior elliptic problems}

The theory of elliptic partial differential equations is far less advanced when these equations are considered on unbounded domains. We give below some examples of physical problems whose mathematical representation leads to free boundary problems in exterior domains (we recall that an exterior domain is the complement of a bounded domain).

An important feature of exterior problems is that they permit us to consider systems of many bodies interacting with each other.

The electrostatics problem. Consider a smooth conducting body $G$ in $\mathbb{R}^{N}(N=2$ or 3$)$ with a charge distribution on its boundary. We recall that 
a charge distribution $\rho \in C(\partial G)$ is called an equilibrium charge distribution if the single-layer potential induced by $\rho$

$$
\psi(x)=\int_{\partial G} \rho(y) \gamma(|x-y|) d \sigma_{y}
$$

is constant in $G$; here $\gamma(t)=-\frac{1}{2 \pi} \log t$ if $N=2$ and $\gamma(t)=-\frac{1}{4 \pi t}$ if $N=3$. Note that the potential $\psi$ is harmonic and smooth in $G$ and $\Omega:=\mathbb{R}^{N} \backslash G$.

We are interested in constant equilibrium charge distributions. First, if $G$ is a ball and $\rho=$ const then $\psi$, being rotationally invariant and harmonic, is constant in $G$. Hence, a natural question is : do non-circular conductors admit constant equilibrium charge distributions ? This question was given a negative answer by Martensen ([14]) and Reichel ([19]), respectively for $N=2$ and $N=3$.

However, the exterior nature of the problem permits an important generalisation, namely, we can ask the same question for several conducting bodies in the space. More precisely, suppose we have $m$ conductors $(m \geq 2)$, with possibly different (but constant !) charge distributions on their boundaries. Can such a system be an equilibrium one? The negative answer is contained in Theorem 4.

The mathematical formulation of the problem is as follows. Suppose we have $C^{2, \alpha}$-regular mutually disjoint bounded domains $G_{1}, \ldots, G_{m}$, such that $\mathbb{R}^{N} \backslash G$ is connected, where

$$
G=\bigcup_{i=1}^{k} G_{i}
$$

Suppose each body $G_{i}$ has a constant equilibrium charge distribution $\rho_{i}$ on its boundary. This means that the single-layer potential defined by (10), with $\rho(y)=\rho_{i}$ for $y \in \partial G_{i}$, is constant in each $G_{i}$.

Then

$$
\frac{\partial \psi}{\partial n}=-\rho_{i} \quad \text { on } \quad \partial G_{i}
$$

by the jump condition for single-layer potentials ; here $n$ is the exterior normal to $\partial G_{i}$ (interior to $\partial \Omega$ ). Furthermore, $\psi$ is always above its value $\psi_{\infty}$ at infinity ; indeed, we have $\psi_{\infty}=-\infty$ for $N=2$ and $\psi_{\infty}=0$ for $N=3$.

Hence, if the system is in equilibrium, then the function $\psi \in C^{2, \alpha}(\bar{\Omega})$ satisfies

$$
\left\{\begin{array}{rlrlrl}
\Delta \psi & =0 & & \text { in } & & \mathbb{R}^{N} \backslash G \\
\psi & >\psi_{\infty} & & \text { in } & \mathbb{R}^{N} \backslash G \\
\psi & =a_{i}>0 & & \text { on } & & \partial G_{i}, i=1, \ldots, m \\
\frac{\partial u}{\partial n} & =-\rho_{i} & & \text { on } & & \partial G_{i}, i=1, \ldots, m
\end{array}\right.
$$


The exterior capillarity problem. Here we consider a large (mathematically speaking : infinite) reservoir full of a homogeneous and incompressible liquid, into which we dip a straight solid cylinder. We study the contact surface between the liquid and the cylinder's wall. This problem is dual to the third problem considered in Section 1.1 and leads to the same equation in the exterior of the cylinder (see [19] and [20]) .

More generally, consider $m$ solid cylindrical bodies of arbitrary (smooth) cross sections $G_{i}, i=1, \ldots, m$, dipped into a large reservoir without touching each other. They make the liquid rise around their walls to some level higher than the (reference) level at the walls of the reservoir. We want to know if the points on the contact surfaces between the liquid and the walls of the cylinders can be at the same height, allowing different heights for the different contact lines. Another way of putting the question is : if we have a set of cylinders dipped into a infinite reservoir, can we build another set of cylinders which, added to the first, will create a system in equilibrium, with each contact surface being at constant height ?

The mathematical problem to which the above question reduces is the following. As in the previous example, suppose we have $m C^{2, \alpha}$-regular mutually disjoint bounded domains $G_{1}, \ldots, G_{m}$, such that $\mathbb{R}^{N} \backslash G$ is connected. We need to investigate the solvability of the problem

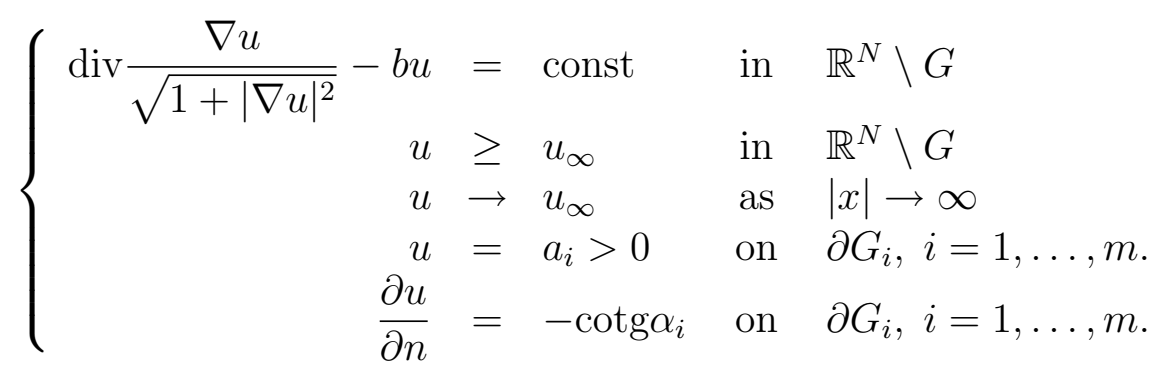

Both (12) and (13) are particular cases of the boundary-value problem considered in Theorem 4 of Section 2. This theorem says (12) and (13) do not have a solution, more precisely, if they do, then $m=1$ and $G=G_{1}$ is a ball. 


\section{The symmetry theorems}

In this section we present the mathematical results which answer the questions posed in Section 1. All of them are based on the famous "moving planes" method of Alexandrov (1962) which has proved to be by far the most powerful tool for establishing symmetry properties of positive solutions of elliptic partial differential equations.

We begin with the case of a bounded domain $\Omega \subset \mathbb{R}^{N}, N \geq 2$. We consider classical (i.e. $C^{2}$-regular) solutions of the problem

$$
\left\{\begin{array}{rlll}
Q u+f(u) & =0 & \text { in } & \Omega \\
u & >0 & \text { in } & \Omega \\
u & =0 & \text { on } & \partial \Omega
\end{array}\right.
$$

where $f \in C^{1}\left(\mathbb{R}^{+}, \mathbb{R}\right)$ and $Q$ is a regular uniformly elliptic operator, that is,

(q) $Q u=\operatorname{div}(g(|\nabla u|) \nabla u)$, where $g \in C^{2}([0, \infty)), g(s)>0$ and $(s g(s))^{\prime}>0$ for all $s \geq 0$.

This assumption is satisfied by the Laplace operator $(Q=\Delta)$, and by the mean curvature operator $\left(Q u=\operatorname{div} \frac{\nabla u}{\sqrt{1+|\nabla u|^{2}}}\right)$. Let us note that all results in this paper remain true for another physically important operator, the MongeAmpère operator $\left(Q u=\operatorname{det}\left(D^{2} u\right)\right)$, and also for any linear uniformly elliptic operator. We note the function $f$ can be allowed to depend on $|\nabla u|$.

We shall often consider (14) together with the following boundary condition ( $n$ will always denote the interior normal to $\partial \Omega$ )

$$
\frac{\partial u}{\partial n}=\text { const } \quad \text { on } \partial \Omega .
$$

We are interested in the following two questions :

- if the domain is symmetric with respect to a hyperplane, do solutions of (14) have the same property?

- if problem (14)-(15) is solvable, then is $\Omega$ a ball ?

These two questions were answered in the affirmative in two classical and very well-known today papers, by Serrin (1971) and Gidas-Ni-Nirenberg (1981).

In the context of elliptic partial differential equations the moving planes method was developed by Serrin in [21], where he proved the following theorem.

Theorem 1 (Serrin, 1971) Suppose $\Omega$ is a bounded $C^{2}$-domain and let $u \in$ $C^{2}(\bar{\Omega})$ be a classical solution of (14)-(15). Then $\Omega$ must be a ball. 
The result of Gidas, Ni and Nirenberg states the following.

Theorem 2 (Gidas-Ni-Nirenberg, 1981) Suppose $\Omega$ is bounded and convex with respect to some direction $\gamma \in \mathbb{R}^{N} \backslash\{0\}$. Suppose also that $\Omega$ is symmetric with respect to a hyperplane perpendicular to $\gamma$. Then any solution of (14) is symmetric with respect to this hyperplane. In addition, all solutions of (14) are strictly decreasing functions along any parallel to $\gamma$ segment, which links the hyperplane and $\partial \Omega$.

In particular, if $\Omega$ is a ball, then any solution of (14) is radial and decreasing, that is, it depends only on, and decreases with, the distance to the center of the ball.

In 1991 Berestycki and Nirenberg (see [6]) gave an alternative proof of this theorem. In this paper they greatly simplified the moving planes method and showed Theorem 2 can be extended to only Liptscitz continuous functions $f$, and to a large class of non-smooth domains.

The Berestycki-Nirenberg improved moving planes method became very popular during the last decade. It was used in many different contexts, where symmetry of solutions of elliptic PDE's was studied.

We next turn to unbounded domains. In $1991 \mathrm{C}$. Li adapted the moving planes method to the case when equation (14) is defined in the whole space. He proved the following theorem.

Theorem 3 (C. Li, 1991) Suppose we have a classical solution of

$$
\left\{\begin{array}{rlll}
Q u+f(u) & =0 & \text { in } & \mathbb{R}^{N} \\
u \geq 0, u & \neq \equiv 0 & \text { in } & \mathbb{R}^{N} \\
u & \rightarrow 0 & \text { as } & |x| \rightarrow \infty,
\end{array}\right.
$$

and, in addition, that $f$ is (weakly) decreasing in a right neighbourhood of zero. Then the solution $u$ is radial with respect to some point $x^{0} \in \mathbb{R}^{N}$, that is, $u$ is a function of $\left|x-x^{0}\right|$ alone, and

$$
\frac{d u}{d r}<0 \quad \text { for } r=\left|x-x^{0}\right| \in(0, \infty) .
$$

In a series of papers Berestycki, Caffarelli and Nirenberg (see [5] and the references therein) studied symmetry properties of positive solutions of elliptic equations in various types of unbounded domains, including a halfspace, a cylinder, and a domain bounded by a Lipschitz graph.

In view of the applications exterior and annuli-like domains were extensively studied too. In particular, a number of important partial results were obtained by Alessandrini, Aftalion-Busca, Willms-Gladwell-Siegel, Phillipin 
and Reichel. None of these results could apply to the problems of many bodies discussed in Section 1.2.

In our forthcoming paper [22] we study these two problems, and answer the questions from Section 1.2. Here is the precise statement of the main result in this paper.

We make the following hypotheses :

(i) we are in the context of Section 1.2, that is, we have a set $G$ which is the union of $m$ mutually disjoint bounded $C^{2, \alpha}$-domains $G_{1}, \ldots, G_{m}$;

(ii) $\quad f$ is a Lipschitz continuous function in $[0, \infty)$ and is decreasing in a right neighbourhood of zero ;

(iii) there exists a $C^{2}$-regular solution of the problem

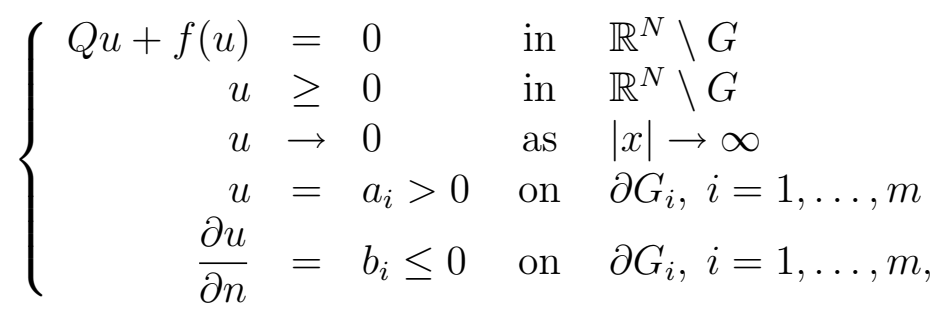

where $a_{i}$ and $b_{i}, i=1, \ldots, m$, are constants and $n$ denotes the exterior normal to the boundary of $G$ (interior to $\partial \Omega=\partial\left(\mathbb{R}^{N} \backslash G\right)$ ).

Remark The constant which appears in the right-hand side of the equation in (13) is necessarily zero, since the solvability of (17) implies $f(0)=0$.

Theorem 4 Suppose (i), (ii) and (iii) hold. Then $m=1, G=G_{1}$ is a ball centered at some point $x^{0} \in \mathbb{R}^{N}$, the solution of (17) $u$ is radial, that is $u=u\left(\left|x-x^{0}\right|\right)$, and

$$
\frac{d u}{d r}<0 \quad \text { for } r=\left|x-x^{0}\right| \in\left(\rho_{1}, \infty\right),
$$

where $\rho_{1}$ denotes the radius of $G$.

There is a version of this theorem for multiply connected bounded domains, more specifically, domains of the type $\Omega \backslash G$, where $\Omega$ is a smooth bounded domain and $G$ is as above. Furthermore, we have a Gidas-NiNirenberg type result in the exterior of a ball. More precisely, if $G$ is a ball and we have a solution of (17), with the last boundary condition relaxed to $\frac{\partial u}{\partial n} \leq 0$ on $\partial G$, then this solution depends only on and decreases with the distance to the center of $G$. 


\section{Maximum principles. The moving planes method in bounded domains}

\subsection{The improved moving planes method.}

In this section we describe the moving planes method of Berestycki and Nirenberg, and give their proof of Theorem 2.

Before going to the method itself we recall two very classical results in elliptic theory, the maximum principle and Hopf's lemma (known also as the strong maximum principle). For their proofs, see for example [17]. More recent (and precise) results are contained in [7].

Theorem 5 (The maximum principle) Let $\Omega$ be a bounded domain and

$$
L=\sum_{i, j=1}^{n} a_{i j}(x) \partial_{i j}+\sum_{i=1}^{n} b_{i}(x) \partial_{i}+c(x)
$$

be a linear uniformly elliptic operator (that is, the matrix $\left(a_{i j}(x)\right)$ is positive definite uniformly in $x)$, with bounded coefficients. Suppose $c(x) \leq 0$ in $\Omega$. Then, for any $u \in C^{2}(\Omega) \cap C^{0}(\bar{\Omega})$,

$$
\left\{\begin{aligned}
L u & \leq 0 \quad \text { in } \quad \Omega \\
u & \geq 0 \text { on } \quad \partial \Omega
\end{aligned}\right.
$$

implies $u \geq 0$ in $\Omega$. We say that $L$ satisfies the maximum principle in $\Omega$.

Theorem 6 (Hopf's lemma) Let $\Omega$ be a bounded domain and $L$ be a linear uniformly elliptic operator with bounded coefficients. Then

$$
\left\{\begin{array}{r}
L u \leq 0 \quad \text { in } \Omega \\
u \geq 0 \text { in } \Omega
\end{array}\right.
$$

implies that either $u \equiv 0$ in $\Omega$ or $u>0$ in $\Omega$ and, in addition, $\frac{\partial u}{\partial n}>0$ on any point of $\partial \Omega$ at which $\partial \Omega$ admits an interior tangent ball and u vanishes.

Note that in Theorem 5 we supposed that the zero-order coefficient of the elliptic operator is non-positive. In general, the maximum principle is false if we do not make a hypothesis on $c(x)$. However, it is possible to give conditions on the domain $\Omega$ which ensure the validity of the maximum principle, for any bounded $c(x)$. In particular, the following maximum principle "in small domains" holds. 
Theorem 7 Let $\Omega$ be a bounded domain, with $\operatorname{diam} \Omega \leq d$. Let $L$ be a linear uniformly elliptic operator with coefficients bounded in the uniform norm by a constant $A$. Then the maximum principle is satisfied by $L$ in $\Omega$, provided $\operatorname{vol}(\Omega)<\delta$, where $\delta$ is a constant depending only on $d, A$, and the ellipticity constant of $L$.

Proof of Theorem 2 Suppose for simplicity $\Omega$ is convex in the direction of the vector $e_{1}=(1,0, \ldots, 0)$ and is symmetric with respect to the hyperplane $T_{0}=\left\{x \mid x_{1}=0\right\}$. We want to show that

$$
u\left(-x_{1}, x_{2}, \ldots, x_{N}\right)=u\left(x_{1}, x_{2}, \ldots, x_{N}\right) \quad \text { for any } x \in \Omega .
$$

For any $\lambda \in \mathbb{R}$ we define

$$
\begin{aligned}
T_{\lambda} & =\left\{x \mid x_{1}=\lambda\right\}, \quad D_{\lambda}=\left\{x \mid x_{1}>\lambda\right\}, \quad \Sigma_{\lambda}=D_{\lambda} \cap \Omega, \\
x^{\lambda} & =\left(2 \lambda-x_{1}, x_{2}, \ldots, x_{n}\right)-\text { the reflexion of } x \text { with respect to } T_{\lambda}, \\
w_{\lambda}(x) & =u\left(x^{\lambda}\right)-u(x), \quad \text { provided } x \in \Sigma_{\lambda}, \\
d & =\inf \left\{\lambda \in \mathbb{R} \mid T_{\mu} \cap \bar{\Omega}=\emptyset \text { for all } \mu>\lambda\right\}
\end{aligned}
$$

(see Fig. 1). With this notation, our goal is to show that $w_{0} \equiv 0$ in $\Sigma_{0}$.

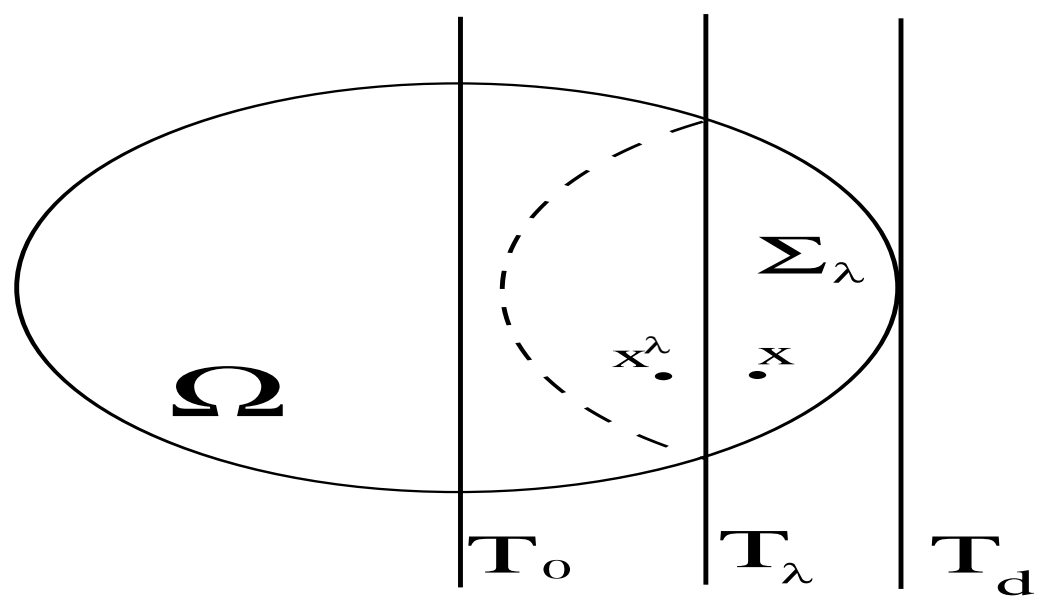

Figure 1: The moving planes method

Lemma 3.1 The function $w_{\lambda}, \lambda \in[0, d)$, satisfies a linear uniformly elliptic equation of the form (summing over repeting indices)

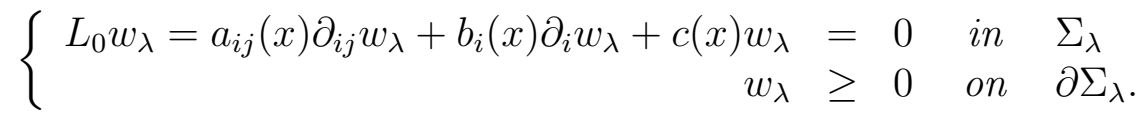

The ellipticity constant and the coefficients of $L$ are bounded independently of $\lambda$. 
This lemma is obvious for $Q=\Delta$ and requires some computations in the case of a more general operator (see for example [20]). Note that we take

$$
c(x)=\left\{\begin{array}{cccc}
\frac{f\left(u\left(x^{\lambda}\right)\right)-f(u(x))}{u\left(x^{\lambda}\right)-u(x)} & \text { if } & u\left(x^{\lambda}\right) \neq u(x) \\
0 & \text { if } & u\left(x^{\lambda}\right)=u(x) .
\end{array}\right.
$$

We say the hyperplane $T_{\lambda}$ has reached a position $\lambda<d$ provided $w_{\mu}$ is non-negative in $\Sigma_{\mu}$, for all $\mu \in[\lambda, d)$. The plane $T_{\lambda}$ "starts" at $\lambda=d$ and "moves" to the left as $\lambda$ decreases. If we prove that $T_{\lambda}$ reaches position zero we are done, since then we can take a hyperplane coming from the other side, that is, starting from $-d$ and moving to the right. The situation is totally symmetric so the second hyperplane would reach position zero too. This means that $w_{0} \geq 0$ and $w_{0} \leq 0$ in $\Sigma_{0}$, hence $w_{0} \equiv 0$ in $\Sigma_{0}$.

Step 1 The above procedure can begin, that is, there exists $\bar{\lambda}<d$ such that $w_{\mu} \geq 0$ in $\Sigma_{\mu}$, for all $\mu \in[\bar{\lambda}, d)$.

Proof By using Theorem 7 we can find a number $\delta$ such that the operator $L_{0}$ defined in Lemma 3.1 satisfies the maximum principle in any subdomain $\Omega^{\prime} \subset \Omega$, with $\operatorname{vol}\left(\Omega^{\prime}\right)<\delta$. We fix $\bar{\lambda}<d$ so close to $d$ that $\operatorname{vol}\left(\Sigma_{\lambda}\right)<\delta$, for any $\lambda \in[\bar{\lambda}, d)$. Hence, by Theorem 7 , equation (18) implies that $w_{\mu} \geq 0$ in $\Sigma_{\mu}$, for all $\mu \in[\bar{\lambda}, d)$.

Note that, by the definition of $w_{\lambda}$, we have $w_{\lambda}>0$ on $\partial \Sigma_{\lambda} \cap \partial \Omega$, for any $\lambda \in(0, d)$ (since $u$ vanishes on $\partial \Omega$ and is strictly positive in $\Omega$ ). Hence, by Hopf's lemma, $w_{\lambda}>0$ in $\Sigma_{\lambda}$, for $\lambda \in(\bar{\lambda}, d)$.

Step 1 permits us to define the number

$$
\lambda_{0}=\inf \left\{\lambda \in(0, d) \mid w_{\mu} \geq 0 \text { in } \Sigma_{\mu} \text { for all } \mu \geq \lambda\right\} .
$$

Note that, by continuity with respect to $\lambda, w_{\lambda_{0}} \geq 0$ in $\Sigma_{\lambda_{0}}$. By Hopf's lemma, if $\lambda_{0}>0$ then $w_{\lambda_{0}}>0$ in $\Sigma_{\lambda_{0}}$.

Step $2 \frac{\partial u}{\partial x_{1}}<0$ in $\Sigma_{\lambda_{0}}$.

Proof Let $x$ be an arbitrary point in $\Sigma_{\lambda_{0}}$, with $x_{1}=\lambda$. Then, by the preceding remarks, $w_{\lambda}>0$ in $\Sigma_{\lambda}$. Since $w_{\lambda}=0$ on $T_{\lambda}$, Hopf's lemma implies

$$
0<\frac{\partial w_{\lambda}}{\partial x_{1}}(x)=-2 \frac{\partial u}{\partial x_{1}}(x)
$$

(recall that $\left.w_{\lambda}(x)=u\left(x^{\lambda}\right)-u(x)\right)$. 
Step $3 \quad \lambda_{0}=0$.

Proof Suppose for contradiction $\lambda_{0}>0$. We are going to "push" the moving plane to the left of $\lambda_{0}$. Let $K$ be a compact subset of $\Sigma_{\lambda_{0}}$ such that

$$
\operatorname{vol}\left(\Sigma_{\lambda_{0}} \backslash K\right)<\frac{\delta}{2}
$$

( $\delta$ is the number from Theorem 7 ). Since $w_{\lambda_{0}}$ is continuous and strictly positive in $\Sigma_{\lambda_{0}}$, there exists a number $\varepsilon>0$ such that $w_{\lambda_{0}} \geq \varepsilon$ in $K$. Fix a number $\lambda_{1}, 0<\lambda_{1}<\lambda_{0}$, such that $\operatorname{vol}\left(\Sigma_{\lambda} \backslash K\right)<\delta$, for $\lambda \in\left[\lambda_{1}, \lambda_{0}\right)$ (see figure 2). By continuity, if $\lambda_{1}$ is sufficiently close to $\lambda_{0}$ we have $w_{\lambda} \geq \frac{\varepsilon}{2}>0$ in $\mathrm{K}$, for

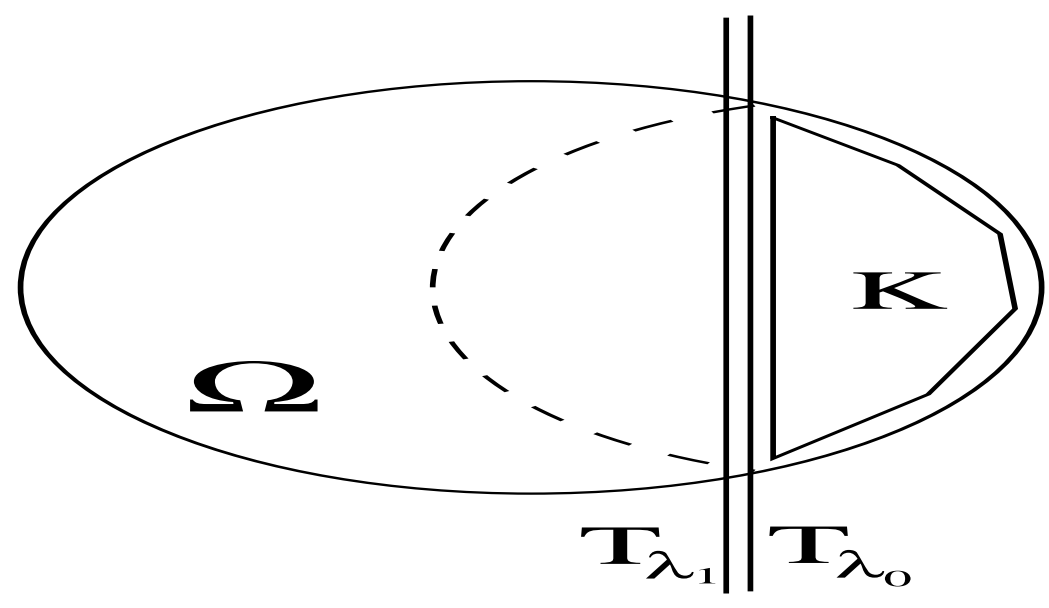

Figure 2: The contradiction in Step 3

any $\lambda \in\left[\lambda_{1}, \lambda_{0}\right)$. In the remaining part of $\Sigma_{\lambda}$ the function $w_{\lambda}, \lambda \in\left[\lambda_{1}, \lambda_{0}\right)$, satisfies the equation

$$
\left\{\begin{aligned}
L_{0} w_{\lambda}=0 & \text { in } \quad \Sigma_{\lambda} \backslash K \\
w_{\lambda_{1}} \geq 0 & \text { on } \quad \partial\left(\Sigma_{\lambda} \backslash K\right) .
\end{aligned}\right.
$$

By Theorem $7, w_{\lambda} \geq 0$ in $\Sigma_{\lambda} \backslash K$. Hence $w_{\lambda} \geq 0$ in $\Sigma_{\lambda}$, for any $\lambda \in\left[\lambda_{1}, \lambda_{0}\right)$. This contradicts the definition of $\lambda_{0}$.

\subsection{Overdetermined Problems. Serrin's lemma}

In this section we give a summary of the proof of Serrin's result (Theorem 1). The idea is to show that for any direction $\gamma \in \mathbb{R}^{N} \backslash\{0\}$ there exists $\lambda=$ $\lambda(\gamma) \in \mathbb{R}$ such that the domain and the solution are symmetric with respect to the hyperplane $T_{\lambda}=\left\{x \in \mathbb{R}^{N} \mid\langle x, \gamma\rangle=\lambda\right\}$; here $\langle\cdot, \cdot\rangle$ denotes the scalar product in $\mathbb{R}^{N}$. 
We fix for instance $\gamma=e_{1}=(1,0, \ldots, 0)$. By using the moving planes method described in the previous section we can show, in exactly the same manner, that a hyperplane starting from position $d$ and moving to the left will move as long as the function $w_{\lambda}$ is defined in $\Sigma_{\lambda}$, that is, as long as the reflexion of $\Sigma_{\lambda}$ with respect to $T_{\lambda}$ is contained in $\Omega$.

In any case the moving plane reaches position $\lambda_{\star}$ (called the critical position), where

$\lambda_{\star}=\inf \left\{\lambda \leq d \mid\left(\Sigma_{\mu}\right)^{\mu} \subset \Omega\right.$ and $<n(z), e_{1}><0$ for all $\left.\mu>\lambda, z \in T_{\mu} \cap \partial \Omega\right\}$

(here, and in the sequel, an upper index means reflexion with respect to the hyperlane with the same index). In other words, the reflexion of $\Sigma_{\lambda}$ with respect to $T_{\lambda}$ stays in $\Omega$ until at least one of the following two events occurs

(i) the reflexion of $\partial \Omega \cap \partial \Sigma_{\lambda}$ wih respect to $T_{\lambda}$ becomes internally tangent to $\partial \Omega$ at some point $P$;

(ii) $T_{\lambda}$ becomes orthogonal to $\partial \Omega$ at some point $Q$.

(see figure 3).

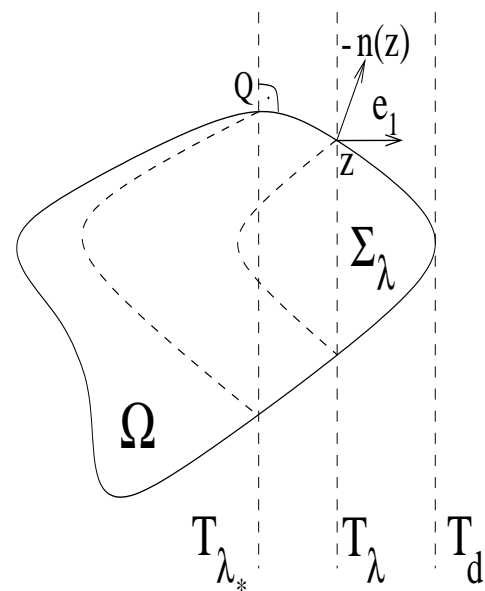

(a)

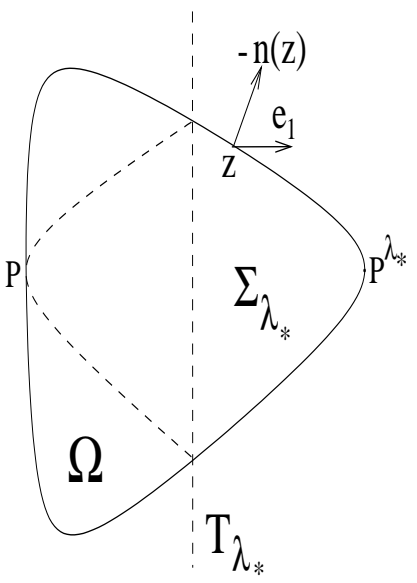

(b)

Figure 3: Two types of domains $\Omega$ : the critical position $\lambda_{\star}$ is attained at a point of orthogonality (a), or at a point of tangency (b). For all $\mu>\lambda_{\star}$ the part of $\bar{\Omega}$ to the right of $T_{\mu}$ has its reflection inside $\Omega$ and the outward normal to $\partial \Omega$ at each point of the boundary of this part makes an acute angle with the direction $e_{1}$.

We are going to show that $w_{\lambda_{\star}} \equiv 0$ in $\Sigma_{\lambda_{\star}}$. Since $u>0$ in $\Omega$, this implies that the reflexion of $\partial \Sigma_{\lambda_{\star}} \cap \partial \Omega$ with respect to $T_{\lambda_{\star}}$ lies on $\partial \Omega$, that is, $\Omega$ is symmetric with respect to $T_{\lambda_{\star}}$. 
In case (i) Hopf's lemma (applied to (18) for $w_{\lambda_{\star}}$ ) immediately yields $w_{\lambda_{\star}} \equiv 0$ in $\Sigma_{\lambda_{\star}}$. Indeed, the function $w_{\lambda_{\star}}$ vanishes at $P^{\lambda_{\star}} \in \partial \Sigma_{\lambda_{\star}} \cap \partial \Omega$ (since $u=0$ on $\partial \Omega$ ) and so does its normal derivative, because of condition (15).

Case (ii) is more difficult to treat, since Hopf's lemma does not apply at $Q\left(\partial \Sigma_{\lambda_{\star}}\right.$ does not admit an interior tangent ball at this point). Serrin proved the following refinement of Hopf's lemma (see [21], p. 313-314). For more general results, see [9].

Lemma 3.2 Let $D^{*}$ be a $C^{2}$-domain and let $T$ be a plane containing the normal to $\partial D^{*}$ at some point $Q$. Let $D$ then denote the portion of $D^{*}$ lying on some particular side of $T$. Suppose $w \in C^{2}(\bar{D})$ satisfies a linear uniformly elliptic inequation with bounded coefficients of the form

$$
\left\{\begin{aligned}
L w=a_{i j}(x) \partial_{i j} w+b_{i}(x) \partial_{i} w & \leq 0 \text { in } D \\
w & \geq 0 \text { in } D .
\end{aligned}\right.
$$

Suppose also that

$$
\left|a_{i j}(x) \xi_{i} \eta_{j}\right| \leq K(|<\xi, \eta>|+|\xi||d(x)|), \quad K=\text { const }
$$

where $\xi \in \mathbb{R}^{N}, \eta \perp T, d(x)=\operatorname{dist}(x, T)$. Under these hypotheses, if $w$ and all its first and second order derivatives vanish at $Q$ then $w \equiv 0$ in $D$.

Let us show that Serrin's lemma applies to $w_{\lambda_{\star}}$, for $Q=\Delta$ and $N=2$ (for the general case we refer the reader to [21], p. 315-316 and [20], Appendix 1). First, $w_{\lambda_{\star}}$ satisfies an equation of type (20), by Lemma 3.1 (we can always achieve $c(x) \geq 0$ in (18), by making the change of functions $\bar{w}=\exp \left(\beta x_{1}\right) w$, with $\beta$ sufficiently large). Recall that

$$
w_{\lambda_{\star}}(x)=u\left(2 \lambda_{\star}-x_{1}, x_{2}\right)-u\left(x_{1}, x_{2}\right), \quad \text { for }\left(x_{1}, x_{2}\right) \in \Sigma_{\lambda_{\star}} .
$$

This trivially yields (we write $w$ instead of $w_{\lambda_{\star}}$ )

$$
\frac{\partial w}{\partial x_{2}}(Q)=\frac{\partial^{2} w}{\partial x_{1} x_{1}}(Q)=\frac{\partial^{2} w}{\partial x_{2} x_{2}}(Q)=0 .
$$

Since $\tau=-e_{1}$ is tangent to $\partial \Omega$ at $Q$ and $n=-e_{2}$ is normal to $\partial \Omega$ at $Q$, we get

$$
\begin{aligned}
\frac{\partial w}{\partial x_{1}}(Q)=-2 \frac{\partial u}{\partial x_{1}}(Q) & =2 \frac{\partial u}{\partial \tau}(Q) \\
\frac{\partial^{2} w}{\partial x_{1} x_{2}}(Q)=-2 \frac{\partial^{2} u}{\partial x_{1} x_{2}}(Q) & =-2 \frac{\partial}{\partial \tau}\left(\frac{\partial u}{\partial n}\right)(Q) .
\end{aligned}
$$

Since both $u$ and its normal derivative are constant on $\partial \Omega$, the last two quantities vanish. By Serrin's lemma $w_{\lambda_{\star}} \equiv 0$ in $\Sigma_{\lambda_{\star}}$. 


\section{Exterior domains. Proof of Theorem 4}

In this section we give a short version of the proof of Theorem 4 . The complete proof is rather lengthy and can be found in [22]. Our goal here is to outline its main ideas.

The proof is based on the moving planes method. The principal difficulties are the following.

- The domain $\Omega$ is unbounded ;

- The domain $\Sigma_{\lambda}$ can be very complex in nature (in particular, not connected, see Fig. 4 below) ;

- We do not know a priori whether the solution is below its value on the boundary $\partial G$.

We shall suppose that

$$
Q=\Delta, \quad m=1, \quad f \in C^{1}\left(\mathbb{R}^{+}\right) \quad \text { and } \quad f^{\prime}(0)<0,
$$

for simplicity. With the notations from the preceding sections, our goal is again to show that the domain $\Omega=\mathbb{R}^{N} \backslash G$ and the solution $u$ are symmetric with respect to a hyperplane $T_{\lambda}$, for some $\lambda$. The function $w_{\lambda}$ is now defined in the set $\Sigma_{\lambda}=D_{\lambda} \backslash \overline{G^{\lambda}}$, for $\lambda \geq \lambda_{\star}$ (here $\lambda_{\star}$ denotes the critical position for $G$; note that $G \subset G^{\lambda}$, for $\lambda \geq \lambda_{\star}$ ).

We divide the proof into ten steps. The first step is again "initializing", in the sense that it permits us to begin the moving plane process.

Step 1 There exists $\bar{\lambda} \in \mathbb{R}$ such that $w_{\lambda} \geq 0$ in $\Sigma_{\lambda}$ for all $\lambda \geq \bar{\lambda}$.

Proof. This step is based on the the proof of C.Li's result (see [13]). We shall take the opportunity to explain its idea. In order to use Li's argument we notice that, since $u$ tends to zero at infinity, we can take $\tilde{\lambda} \in \mathbb{R}$ such that $\tilde{\lambda}>d$ and

$$
u(x)<\frac{a_{1}}{2} \quad \text { for } \quad|x|>\tilde{\lambda},
$$

so that $w_{\lambda}>\frac{a_{1}}{2}>0$ on $\partial G^{\lambda}$, for all $\lambda \geq \tilde{\lambda}$.

Suppose the claim in Step 1 is false, that is, there exists a sequence $\left\{\lambda_{m}\right\}_{m=1}^{\infty}$ such that

$$
\lim _{m \rightarrow \infty} \lambda_{m}=\infty, \lambda_{m} \geq \tilde{\lambda}
$$

and $w_{\lambda_{m}}$ takes negative values in $\Sigma_{\lambda_{m}}$. Since $w_{\lambda}$ is zero on $T_{\lambda}$ and tends to zero at infinity for a fixed $\lambda$, we see that $w_{\lambda_{m}}$ attains its negative minimum in the interior of $\Sigma_{\lambda_{m}}$, say at a point $x^{(m)}$. Then

$$
\nabla w_{\lambda_{m}}\left(x^{(m)}\right)=0 \quad \text { and } \quad \Delta w_{\lambda_{m}}\left(x^{(m)}\right) \geq 0 .
$$


Recall that $w_{\lambda}$ satisfies a linear equation of the type

$$
\Delta w_{\lambda}+c_{\lambda}(x) w_{\lambda}=0 \quad \text { in } \Sigma_{\lambda}
$$

for all $\lambda \in \mathbb{R}$, where $c_{\lambda}(x)=f^{\prime}(d(\lambda, x))$, with

$$
d(\lambda, x) \in\left[\min \left\{u\left(x^{\lambda}\right), u(x)\right\}, \max \left\{u\left(x^{\lambda}\right), u(x)\right\}\right]
$$

(see (19)). Since $w_{\lambda_{m}}\left(x^{(m)}\right)<0$ we see that $0 \leq d\left(\lambda_{m}, x^{(m)}\right) \leq u\left(x^{(m)}\right)$ and therefore $\lim _{m \rightarrow \infty} d\left(\lambda_{m}, x^{(m)}\right)=0$. It follows that $c_{\lambda_{m}}\left(x^{(m)}\right)$ is strictly negative for $m$ large. Hence

$$
0 \leq \Delta w_{\lambda_{m}}\left(x^{(m)}\right)=-c_{\lambda_{m}}\left(x^{(m)}\right) w_{\lambda_{m}}\left(x^{(m)}\right)<0,
$$

a contradiction.

Step 1 shows that the number

$$
\lambda_{0}=\inf \left\{\lambda \in \mathbb{R} \mid w_{\mu} \geq 0 \text { in } \Sigma_{\mu} \text { for all } \mu>\lambda\right\}
$$

is well defined. It is obvious that $\lambda_{0}$ is finite. Notice that, by continuity, $w_{\lambda_{0}} \geq 0$ in $\Sigma_{\lambda_{0}}$.

Step 2 We have $\frac{\partial u}{\partial x_{1}}<0$ in the set $\left\{x \in \mathbb{R}^{N} \mid x_{1}>\max \left\{\lambda_{0}, d\right\}\right\}$.

The proof of this step is similar in idea to the proof of Step 2 in Section 3.1.

Step $3 \lambda_{0} \leq d$.

The ideas of the proof of this step appear in the proof of Step 7 below.

Step 4 For any $z \in \partial G$ and any unit vector $\eta$, for which $<\eta, n(z)>>0$, we can find a sufficiently small ball $B_{\delta}(z)$ such that

$$
\frac{\partial u}{\partial \eta}(\zeta)<0 \quad \text { for all } \zeta \in B_{\delta}(z) \backslash \bar{G} .
$$

Proof. Step 4 claims that the solution $u$ is strictly decreasing in a neighbourhood of $\partial G$, along any outgoing direction. If $\alpha_{1}<0$, this claim is obvious, by continuity. Hence we can assume $\alpha_{1}=0$, or equivalently $\nabla u \equiv 0$ on $\partial G$. Notice that this implies $\left|D^{2} u\right|=\left|\frac{\partial^{2} u}{\partial n^{2}}\right|$ on $\partial G$.

Fix a point $z^{0} \in T_{d} \cap \partial G$, so that

$$
\frac{\partial u}{\partial x_{1}}\left(z^{0}\right)=\frac{\partial u}{\partial n}\left(z^{0}\right)=0
$$


(note that $n=e_{1}$ at $z_{0}$ ). Steps 2 and 3, together with the assumption $\lambda_{0} \leq d$, imply

$$
\frac{\partial u}{\partial x_{1}}\left(z^{0}+t e_{1}\right)<0
$$

for positive $t$. We conclude that

$$
\frac{\partial^{2} u}{\partial n^{2}}\left(z^{0}\right)=\frac{\partial^{2} u}{\partial x_{1}^{2}}\left(z^{0}\right) \leq 0 .
$$

On the other hand, it is easy to compute that $u=$ const and $\nabla u=0$ on $\partial G$ imply

$$
\left.\Delta u\right|_{\partial G}=\left.\frac{\partial^{2} u}{\partial n^{2}}\right|_{\partial G} .
$$

Hence

$$
\frac{\partial^{2} u}{\partial n^{2}} \equiv-f\left(a_{1}\right)=\text { const } \quad \text { on } \partial G .
$$

By (22), $f\left(a_{1}\right) \geq 0$. If $f\left(a_{1}\right)>0$, Step 4 follows easily, since

$$
\frac{\partial^{2} u}{\partial \eta^{2}}=<\eta, n>^{2} \frac{\partial^{2} u}{\partial n^{2}} \quad \text { on } \partial G .
$$

If $f\left(a_{1}\right)=0$, we see that all first and second order derivatives of $u$ vanish on $\partial G$. This implies that the function

$$
\bar{u}(x)=\left\{\begin{array}{cll}
u(x) & \text { for } & x \in \mathbb{R}^{N} \backslash G \\
a_{1} & \text { for } & x \in \bar{G}
\end{array}\right.
$$

belongs to $C^{2}\left(\mathbb{R}^{N}\right)$ and solves the equation

$$
\left\{\begin{array}{llc}
\Delta \bar{u}+f(\bar{u})=0 & \text { in } & \mathbb{R}^{N} \\
\bar{u} \geq 0, \quad \bar{u} \not \equiv 0 & \text { in } & \mathbb{R}^{N} \\
\bar{u}(x) \rightarrow 0 & \text { as } & x \rightarrow \infty
\end{array}\right.
$$

However, the shape of $\bar{u}$ contradicts C. Li's result for equations on $\mathbb{R}^{N}$ (Theorem 3).

Step $5 w_{\lambda}>0$ in $\Sigma_{\lambda}$, for any $\lambda \in\left[\lambda_{0}, \infty\right) \cap\left(\lambda_{\star}, \infty\right)$.

Step 6 We have

$$
\frac{\partial u}{\partial x_{1}}<0 \quad \text { in } D_{\lambda^{\star}} \backslash \bar{G}
$$

where $\lambda^{\star}=\max \left\{\lambda_{0}, \lambda_{\star}\right\}$. 


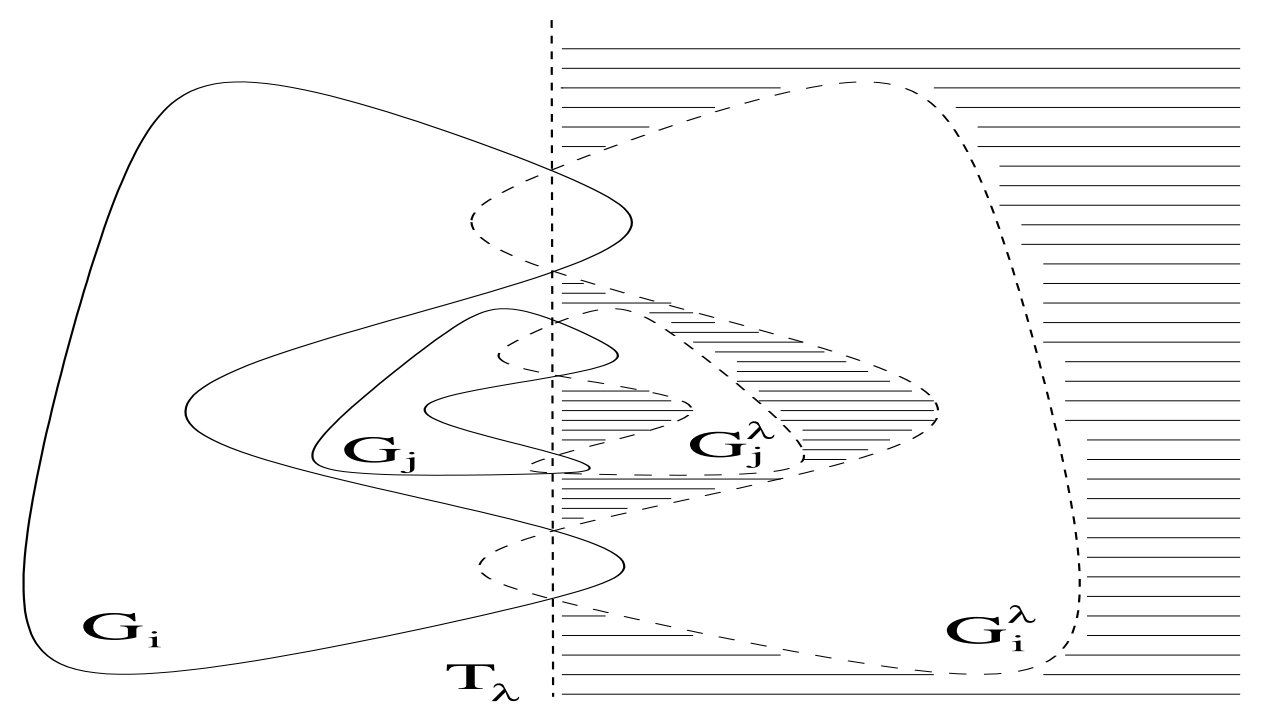

Figure 4: The shaded regions are the connected components of $\Sigma_{\lambda}$.

The last two steps follow from Hopf's lemma. However, we have to be careful about the fact that the domain $\Sigma_{\lambda}$ may have many connected components (see Figure 4 ), so we have to exclude $w_{\lambda} \equiv 0$ in each of them. Suppose for contradiction that $w_{\lambda} \equiv 0$ in a connected component $Z$ of $\Sigma_{\lambda}$. By Step 3 we can suppose $\lambda \leq d$. We observe that the boundary of each connected component of $\Sigma_{\lambda}$ contains a point of intersection between $\partial G$ and $T_{\lambda}$. We take a point $y \in Z$ so close to such a point of intersection $z \in \bar{Z}$, that $y$ belongs to the ball $B_{\delta}(z)$ defined in Step 4 . Then, by Step 4, $u$ decreases strictly from $y^{\lambda}$ to $y$. Hence $w_{\lambda}(y)>0$, a contradiction.

The next step is the most involved part of the proof of Theorem 4. We shall avoid being too rigorous here, and try to concentrate on the main ideas.

Step $7 \lambda_{0}=\lambda_{\star}$.

Proof. According to the defintion of $\lambda_{0}$, we can find two sequences $\left\{\lambda_{m}\right\}_{m=1}^{\infty}$, $\left\{x^{(m)}\right\}_{m=1}^{\infty}$, such that

$$
\lim _{m \rightarrow \infty} \lambda_{m}=\lambda_{0}, \lambda_{\star}<\lambda_{m}<\lambda_{0}, x^{(m)} \in \overline{\Sigma_{\lambda_{m}}} \backslash T_{\lambda_{m}}
$$

and $w_{\lambda_{m}}$ attains its negative minimum in the closure of $\Sigma_{\lambda_{m}}$ at $x^{(m)}$.

A number of different situations may arise. We obtain a contradiction in each of them.

Case 1 There is a subsequence of $\left\{x^{(m)}\right\}$, such that $x^{(m)} \in \operatorname{int} \Sigma_{\lambda_{m}}$. 
We know that $\Delta w_{\lambda_{m}}\left(x^{(m)}\right) \geq 0$ and $\nabla w_{\lambda_{m}}\left(x^{(m)}\right)=0$. If $\lim _{m \rightarrow \infty}\left|x^{(m)}\right|=\infty$ we obtain a contradiction as in Step 1. If a subsequence of $\left\{x^{(m)}\right\}$ converges to a point $x^{0}$ which belongs to $\Sigma_{\lambda_{0}}$ or to the regular part of $\partial \Sigma_{\lambda_{0}}$, by passing to the limit we obtain $w_{\lambda_{0}}\left(x^{0}\right) \leq 0$ and $\nabla w_{\lambda_{0}}\left(x^{0}\right)=0$. This implies that $x^{0}$ belongs to $\partial \Sigma_{\lambda_{0}}$ and $w_{\lambda_{0}}\left(x^{0}\right)=0$, so Hopf's lemma yields $\nabla u\left(x^{0}\right) \neq 0$, a contradiction. If a subsequence of $\left\{x^{(m)}\right\}$ converges to a point $x^{0}$ which belongs to the singular part of $\partial \Sigma_{\lambda_{0}}$ (which is nothing else but $\partial G \cap T_{\lambda_{0}}$ ), then, as in the proof of Steps 5-6, we get $w_{\lambda_{m}}\left(x^{(m)}\right)>0$ for large $m$, which is a contradiction.

Case 2 There is a subsequence of $\left\{x^{(m)}\right\}$, such that $x^{(m)} \in \partial \Sigma_{\lambda_{m}}$.

The following lemma plays a crucial role.

Lemma 4.1 Suppose $\lambda \geq \lambda_{\star}$. Any $z \in \partial G^{\lambda} \cap D_{\lambda}$ has one of the following properties

(I) If we move along direction $-e_{1}$, from $z$ to the left, we enter $\Sigma_{\lambda}$;

(II) If we move along direction $-e_{1}$, from $z$ to the left, we enter $G^{\lambda}$ and, in addition, we meet $\partial G^{\lambda}$ again before or on reaching $T_{\lambda}$;

(III) If we move along direction $-e_{1}$, from $z$ to the left, we enter $G^{\lambda}$ and, in addition, we meet $\partial G$ before or on reaching $T_{\lambda}$;

(IV) $\lambda=\lambda_{\star}$ and $z \in \partial G^{\lambda} \cap \partial G$ (the symmetry case).

The four cases of Lemma 4.1 are shown on Fig. 5. In this way we obtain four types of points on $\partial G^{\lambda} \cap D_{\lambda}$.

Since $\lambda_{m}>\lambda_{\star}$ all points $x^{(m)}$ are of one of the first three types. To simplify the notation we omit the index $m$. Suppose $x$ is of type (I). Then, by Step $6, \frac{\partial u}{\partial\left(-e_{1}\right)}(x)$ is strictly positive. By the boundary conditions in (17) and the fact that $e_{1}$ is an outgoing direction with respect to $\partial G$ at $x^{\lambda}$, we have $\frac{\partial u}{\partial e_{1}}\left(x^{\lambda}\right) \leq 0$. This yields $\frac{\partial w_{\lambda}}{\partial\left(-e_{1}\right)}(x)<0$, which contradicts the minimal choice of $x$.

Suppose next that $x$ is of type (II) or (III). Suppose for simplicity that, when we move along $-e_{1}$ to the left of $x$, the first point $\bar{x} \in \partial G \cup \partial G^{\lambda}$ we reach is to the right of $T_{\lambda_{0}}$. Then, by Step $6, u(\bar{x})>u(x)$. If $x$ is of type (II) we obtain

$$
w_{\lambda}(\bar{x})=a_{1}-u(\bar{x})<a_{1}-u(x)=w_{\lambda}(x),
$$

which contradicts the minimal choice of $x$. If $x$ is of type (III) we obtain $a_{1}=u(\bar{x})>u(x)$. Hence $w_{\lambda}(x)=a_{1}-u(x)>0$, which is a contradiction (recall that $x$ was chosen so that $w_{\lambda}(x)<0$ ). 


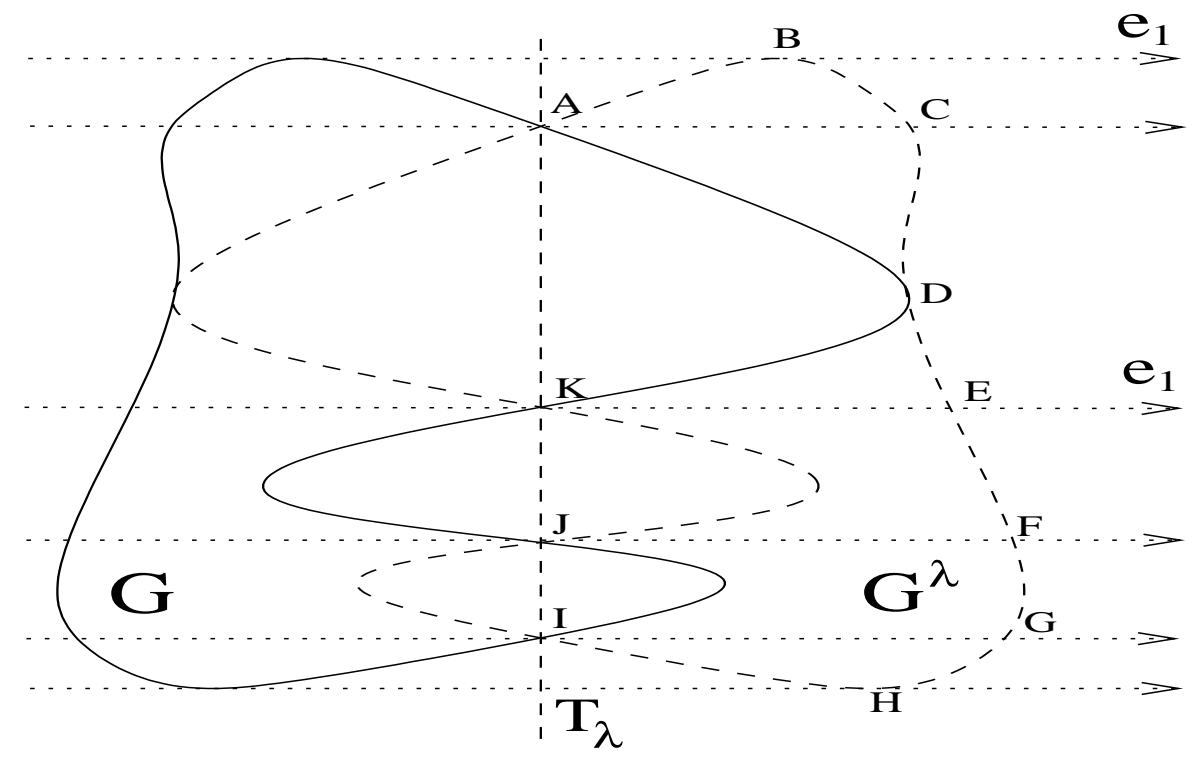

Figure 5: Four types of points on $\partial G^{\lambda} \cap D_{\lambda}$ : the arcs $(A, B],[H, I),(J, K)$ are of type (I), the arcs $(B, C],[E, F],[G, H)$ are of type (II), the arcs $(C, D),(D, E)$, $(F, G)$ are of type (III), and the point $D$ is of type $(I V)$.

Step $8 w_{\lambda_{\star}} \equiv 0$ in at least one connected component of $\Sigma_{\lambda_{\star}}$.

This is a consequence of Hopf's lemma and Serrin's lemma (see Section 3.2).

Step 9 Let $Z$ be a connected component of $\Sigma_{\lambda_{\star}}$ such that $w_{\lambda_{\star}} \equiv 0$ in $Z$. Then

$$
\partial Z \backslash T_{\lambda_{\star}} \subset \partial G
$$

Step 9 says all points on $\partial Z \backslash T_{\lambda_{\star}}$ are of the symmetry type (IV). The other three types of points are excluded by an argument similar to the one we used in the proof of Step 7.

Step 10 Conclusion.

Once we have proved Step 9, the conclusion is obtained via a (not quite explicit) topological argument, due to Fraenkel.

\section{References}

[1] Alessandrini G. A symmetry theorem for condensers. Math. Meth. Appl. Sc. 15:315-320, (1992). 
[2] Alexandrov A.D. A characteristic property of the spheres. Ann. Mat. Pura Appl. 58:303-354, (1962).

[3] Amick C.J. and Fraenkel L.E. Uniqueness of Hill's spherical vortex. Arch. Rat. Mech. Anal. 92:91-119, (1986).

[4] Aftalion A. and Busca J. Radial symmetry for overdetermined elliptic problems in exterior domains. Arch. Rat. Mech. Anal. 143:195-206, (1998).

[5] H. Berestycki, L. Caffarelli, L. Nirenberg Further qualitative properties for elliptic equations in unbounded domains. Dedicated to Ennio De Giorgi. Ann. Scuola Norm. Sup. Pisa Cl. Sci. 25 (1997), no. 1-2, 69-94 (1998).

[6] Berestycki H. and Nirenberg L. On the method of moving planes and the sliding method. Bull. Soc. Brazil Mat. Nova Ser. 22:1-37, (1991).

[7] H. Berestycki, L. Nirenberg, S.R.S. Varadhan The principal eigenvalue and maximum principle for second order elliptic operators in general domains, Comm. Pure Appl. Math. 47 (1994).

[8] Castro A. and Shivaji R. Non-negative solutions to a semilinear Dirichlet problem in a ball are positive and radially symmetric. Comm. Part. Diff. Eq. 14(8\&9):1091-1100, (1989).

[9] Fraenkel L. An introduction to maximum principles and symmetry in elliptic problems. Cambridge Tracts in Mathematics, 128. Cambridge University Press, Cambridge, 2000.

[10] Gidas B., Ni W.-M. and Nirenberg L. Symmetry and related properties via the maximum principle. Comm. Math. Phys. 6:883-901, (1981).

[11] Gilbarg D. and Trudinger N. Elliptic partial differential equations of second order. $2^{\text {nd }}$ edition. Springer-Verlag. (1983).

[12] Landau L.D. and Lifschitz E.M. Fluid Mechanics Pergamon Press (English translation), (1966). 9

[13] Li C. Monotonicity and symmetry of solutions of fully nonlinear elliptic equations on unbounded domains. Comm. Part. Diff. Eq. 16:585-615, (1991).

[14] Martensen E. Eine Integralgleichung für die log. Gleichgewichtsverteilung und die Krümmung der Randkurve eines ebenen Gebiets. Z. Angew. Math. Mech. (72): T596-T599, (1992).

[15] Myshkis A.D. And AL. Low gravity fluid mechanics. Mathematical theory of capillary phenomena, Springer-Verlag (English translation), (1987).

[16] Philippin G.A. On a free boundary problem in electrostatics. Math. Meth. Appl. Sc. 12:387-392, (1990). 
[17] M.H. Protter, H.F. Weinberger Maximum principles in differential equations, Springer-Verlag, New York-Berlin, (1984).

[18] ReIcheL W. Radial symmetry by moving planes for semilinear elliptic BVP's on annuli and other non-convex domains. In Progress in PDE's: Elliptic and Parabolic problems (Pitman Res. Notes, Vol. 325; eds: C.Bandle and al), pp. 164-182

[19] ReicheL W. Radial symmetry for elliptic boundary-value problems on exterior domains. Arch. Rat. Mech. Anal. 137:381-394, (1997).

[20] Reichel W. Radial symmetry for an electrostatic, a capillarity and some fully nonlinear overdetermined problems on exterior domains. Z. Anal. Anwendungen 15:619-635, (1996).

[21] Serrin J. A symmetry theorem in potential theory. Arch. Rat. Mech. Anal. 43:304-318, (1971).

[22] Sirakov B. Exterior elliptic problems and two conjectures in potential theory. To appear in Ann. Inst. Henri Poincaré.

[23] Sokolnikoff I.S. Mathematical theory of elasticity, McGraw-Hill, (1956).

[24] Willms N.B., Gladwell G. and Siegel D. Symmetry theorems for some overdetermined boundary-value problems on ring domains. Z. Angew. Math. Phys. 45:556-579, (1994). 